\title{
Topic: EXPERIMENTAL SURGERY
}

\section{PO:44 \\ MACROPOROUS MESHES WITH ADHESION BAR- RIER: EXPERIMENTAL COMPARATIVE STUDIES IN IPOM VENTRAL HERNIA REPAIR}

F. Ceci ${ }^{1}$, S. Mattia' ${ }^{1}$, E. Manzi ${ }^{1}$, L. D'Amore ${ }^{1}$, F. Gossetti ${ }^{1}$, P. Negro ${ }^{1}$

${ }^{\prime}$ Dept. of surgery P.Stefanini - Sapienza University, Rome, ITALY

Introduction: The increasing use of meshes in abdominal wall hernia repair led to a raising number of postoperative complications, especially due to adhesion formation. Different strategies have been designed to minimize this problem, including the development of synthetic prosthetic materials with antiadhesive layer, mainly in intraperitoneal repair (laparoscopy). We hereby report an experimental study we performed to test the adhesion formation of four different antiadhesive prostheses in a rat ventral hernia model

Materials and methods: Fifty-two mature, male Sprague-Dawley rats weighing 270-365 gr. were obtained from Harlan Laboratories s.r.l. (S.Pietro, Udine, Italy). The animal study was officially approved in accordance with the animal protection law (Research project under the Legislative Decree 116/92 Ministry of Health). Four antiadhesive prostheses were evaluated in 4 groups of rats (13 rats each). The comparative assessment regards synthetic macroporous materials equipped with anti adhesion technology.

The meshes used were the following:

- DynaMesh ${ }^{\circledR}$-IPOM (Distrex Italy): PVDF/PP (Group 1)

- DynaMesh $($ )- CICAT (FEG Textiltechnik): PVDF (Group 2)

- C-Qur/FX (Atrium Medical): PP/Omega 3 (Group 3)

- TiMesh $囚$ (GFE Medizintechnik- PFM Medical): PP/Titanium (Group 4)

The model involved the removal of a $2 \times 2 \mathrm{~cm}$ segment of full-thickness ventral abdominal wall from the midline. The defect was replaced with a $3 \times 3 \mathrm{~cm}$ piece of one of four prostheses, fixed to the anterior layer of the muscles by glue ( $n$-butyl-2-cyanoacrilate, Glubran ${ }^{\circledR} 2$; GEM S.r.1.). Autopsies were performed 7 or 21 days after operation. Adhesion formation was macroscopically scored and extent, severity and required dissection were assessed using the Adhesion Grading Score (AGS) and the Total Adhesion Score (TAS) was determined. Results were analyzed with ANOVA (Tukey.Kramer test-significativity $\mathrm{P}<0.05$ ).

Results: Two rats died within 48 hours after surgery for anesthesia (Group 1). All others animals lived throughout the study. Results are showed in Tab.1
Adhesions were observed in all groups except in some cases in Group 3 at 7 days and Group 4 at 21 days. At 7 days, only $20 \%$ showed no adherences. TiMesh $\left({ }^{\circledR}\right.$ and CICAT showed a larger extension of adherence ( $49 \%$ and $43 \%$ respectively). The results at 21 days changed in each group. The greater extention of adhesions was in CICAT group (45\%). CQur/FX and TiMesh (B) demonstrated best results in terms of required dissection.

Conclusion: All of the tested prostheses attracted adhesions but the antiadhesive barrier reduces the areas of adhesion depending on the physical structure of the mesh (porosity and texture). The intraperitoneal use of Cicat must be avoided, according to care by the Company. The PP component of DynaMesh $(\mathbb{B}$-Ipom reduces its antiadhesive properties. Best results in terms of antiadhesivity are provided by TiMesh $($ and C-Qur/ FX.

Based on these evidences and literature, some suggestions can be pointed out. Prostheses with antiadhesive layer (laminar) are indicated for intraperitoneal use, such as laparoscopic repair. Macroporous materials with antiadhesive barrier are strongly suggested in open repair, according to Rives-Stoppa technique, due to their good tissue ingrowth and limited inflammatory reaction.

\begin{tabular}{|c|c|c|c|c|c|}
\hline & & AGS & AGS & AGS & \\
\hline Mesh & Day & Extension & Severity & $\begin{array}{l}\text { Required } \\
\text { dissection }\end{array}$ & TAS \\
\hline \multirow[t]{2}{*}{ TiMesh@8 } & 7 & 2,2 & 2,6 & 2,8 & 7,6 \\
\hline & 21 & 1,25 & 1,5 & 2,1 & 4,9 \\
\hline \multirow[t]{2}{*}{ IPOM } & 7 & 1,8 & 1,2 & 2,2 & 5,2 \\
\hline & 21 & 1,8 & 1,8 & 2,2 & 5,8 \\
\hline \multirow[t]{2}{*}{ CICAT } & 7 & 2 & 2,2 & 2,8 & 7 \\
\hline & 21 & 2,25 & 2,25 & 2,6 & 7,1 \\
\hline \multirow[t]{2}{*}{ CQur ${ }^{\mathrm{TM}} / \mathrm{FX}$} & 7 & 1,2 & 1 & 1 & 3,2 \\
\hline & 21 & 1,5 & 1,5 & 1,9 & 4,9 \\
\hline
\end{tabular}

Tabie $1:$ AGS mean values and TAS 